ORIGINAL ARTICLE

\title{
Rate of metoclopramide infusion affects the severity and incidence of akathisia
}

\author{
I Parlak, R Atilla, M Cicek, M Parlak, B Erdur, M Guryay, M Sever, S Karaduman
}

Emerg Med J 2005;22:621-624. doi: 10.1136/emj.2004.014712

See end of article for authors' affiliations

.....................

Correspondence to: Dr R Atilla, Dokuz Eylul University Hospital,

Department of Emergency Medicine, Inciralti 35340 Izmir, Turkey; ridvan.atilla@

deu.edu.tr

Accepted for publication 22 August 2004

\begin{abstract}
Objective: To investigate the effect of the rate of metoclopramide infusion on akathisia incidence, severity, onset of symptoms, and duration in patients with headache, and/or nausea/vomiting in the emergency department (ED) setting.

Methods: Prospective, double blind, randomised clinical study comparing two rates of intravenous infusion of metoclopramide over a period of six months at a tertiary university hospital ED.

Results: A total of 300 patients presented to the ED met the inclusion criteria: 151 (50.3\%) with nausea/ vomiting, $108(36 \%)$ with headache, and $41(13.7 \%)$ with headache and nausea/vomiting. Of these, 154 patients (51.3\%) were given $10 \mathrm{mg}$ metoclopramide as a slow intravenous infusion over 15 minutes plus placebo (SIG group) and 146 patients were given $10 \mathrm{mg}$ metoclopramide intravenous bolus infusion over two minutes plus placebo (BIG group). Nine of the 154 patients in the SIG group (5.8\%) had akathisia compared with $36 / 146$ patients $(24.7 \%$ ) in the BIG group ( $p<0.001$, OR $5.273,95 \% \mathrm{Cl} 2.43$ to 11.403 ). Severe akathisia were observed in 13/45 (28.8\%). The incidence of severe akathisia was significantly higher in the BIG group $(30.5 \% ; 11 / 36)$ than in the SIG group $(22.2 \% ; 2 / 9), p=0.009$. Metoclopramide successfully relieved the presenting symptom(s) of 137/146 (90.8\%) and 139/154 (90.2\%) patients in the BIG and SIG groups, respectively.

Conclusions: This study suggests that slowing the rate of infusion of metoclopramide is an effective strategy for reducing the incidence of akathisia in patients with headache, and/or nausea/vomiting in ED.
\end{abstract}

$\mathrm{T}$ he term "akathisia" describes a syndrome of objective and subjective motor restlessness manifested as the inability to sit or stand still. Clinically, akathisia can be described as the urge to move the extremities or torso and anxiety with agitation. The movements are typically stereotypical motor patterns such as pacing, body rocking, or foot tapping. Akathisia also occurs in some disorders of the basal ganglia including drug induced movement disorders. It is used to describe the restlessness observed in patients secondary to neuroleptic drug use, typically manifested by excessive voluntary movement. Dopamine blocking agents such as antipsychotics and antiemetics may induce akathisia. ${ }^{1}$ However, the phenomenon was observed before the introduction of antipsychotics, and the term "akathisia" was used in 1901 by Ladislav Haskovec, a Czech neurologist. ${ }^{2}$

Metoclopramide is a benzamide antiemetic drug, frequently used in emergency departments (EDs) in the management of nausea, vomiting, and vascular-type headache..$^{3-6}$ The US Food and Drug Administration has limited its indications to diabetic gastroparesis, oesophageal reflux, gastrointestinal radiography, intestinal intubation, and nausea/vomiting. The literature indicates that there may be a direct relation between serum concentration of metoclopramide and akathisia, and that slow infusion of metoclopramide could reduce the akathisia. ${ }^{78}$ Akathisia can easily be missed by physicians in crowded and busy EDs. If the initial symptoms are underdiagnosed, massive anxiety and agitation can lead to refusal of treatment and patients pulling out their intravenous lines, leaving the ED, and even attempting suicide. ${ }^{9-13}$

We carried out this study because a literature search (Pub Med accessed on 7 November 2002; keywords: Akathisia, Metoclopramide, Akathisia and metoclopramide, migraine headache and treatment, nausea) revealed a lack of double blind, randomised clinical trials investigating the effects of metoclopramide infusion rates on the incidence of akathisia.

\section{METHODS}

\section{Study design}

We conducted a prospective, double blind, randomised clinical study to compare the effects of:

(a) a fast intravenous infusion of metoclopramide over two minutes plus a slow infusion of placebo over 15 minutes

and

(b) a slow intravenous infusion of metoclopramide over 15 minutes plus fast infusion of placebo over two minutes

with regard to the incidence, severity, onset of symptoms, and the duration of akathisia. The main outcome measure of our study was the proportion of study participants in each group with akathisia at 60 minutes. The Dokuz Eylul University Hospital Review Board approved the study. All the participants signed an informed consent form before enrolment.

\section{Study population and setting}

The study was conducted between July and December 2001 at the Dokuz Eylul University Hospital Emergency Department, which has an annual rate of 36000 visits. The inclusion criteria were: age 17 years or older and any indication for metoclopramide, such as headache and/or nausea/vomiting. In addition, patients could have been taking other medications (which were otherwise included in the exclusion criteria) for added relief of symptoms. The exclusion criteria were: initial akathisia score 3 or greater, previous organic brain disorder, dementia, severe agitation or anxiety that required rescue medication and did not allow interpretation of akathisia symptoms (severe renal colic, acute myocardial infarction, severe nausea/vomiting, or acute gastrointestinal bleeding), pregnancy, lactation, previous 
neurological motor diseases (restless leg syndrome or Parkinson's disease), any contraindication for anticholinergic medications (glaucoma, urinary retention, or bowel obstruction), recent administration of anticholinergic, sedative, antiakathisic, antiemetic, antihistaminic, antipsychotic, antispasmodic drugs within the last three days, and antidepressants, lithium, barbiturates, benzodiazepines, other sedativehypnotics and opioid drugs within the last two weeks.

\section{Study protocol}

All patients eligible for the study were randomised to one of two groups:

- BIG-10 mg metoclopramide in an intravenous bolus infusion over two minutes plus placebo (100 ml normal saline in a slow infusion over 15 minutes)

- SIG-10 mg metoclopramide in $100 \mathrm{ml}$ normal saline given as a slow intravenous infusion over 15 minutes plus placebo ( $2 \mathrm{ml}$ normal saline in a bolus infusion over two minutes)

Akathisia scores were recorded at 0, 5, 15, 30, and 60 minutes according to the Prince Henry Hospital Rating Scale of Akathisia. ${ }^{14}$ Patient satisfaction and side effects other than akathisia (allergic reactions, hypotension, hypertension, dystonic reactions, sedation, etc.) were also recorded at the end of 60 minutes.

At 60 minutes we discontinued observation for akathisia and further management of the patients was planned by their primary physician in the ED. Anxiolytic agents or diphenhydramine were prescribed for patients who had akathisia scores greater than 13 (major akathisia) or if a patient requested a rescue medicine regardless of their total score during the study period. The patients' presenting symptom(s) were evaluated after one hour and a 50\% reduction in the severity of the symptoms was accepted as successful.

\section{Measures}

We used the Prince Henry Hospital Rating Scale of Akathisia (PHH Akathisia Scale) (table 1). ${ }^{14}$ The PHH akathisia scale has objective and subjective components rated between 0 and 3 (from absent to severe, respectively). The sum total of the components (global rating) gives the $\mathrm{PHH}$ akathisia score: absent (0), mild (1), moderate (2), or severe (3). The objective components were rated by the observer and the subjective components were rated based on the response from the patient to the questions. The data were collected by emergency medicine residents other than the physicians providing treatment in the ED. They were all senior residents (postgraduate year 4) in the hospital's emergency medicine residency programme who had attended a two hour training course in data collection, $\mathrm{PHH}$ akathisia scale rating, clinical diagnosis, and management of akathisia, prior to commencement of patient enrolment.

\section{Data analysis}

We analysed the data with SPSS 11.0 for Windows; the $\chi^{2}$ test was used for analysing the demographic characteristics of both groups and the $t$ test was used for categorical data and determination of akathisia incidence. A p value $<0.05$ was accepted as statistically significant and $95 \%$ confidence intervals (CI) for the differences were calculated. With regard to sample size, it was calculated that least 94 patients should be included in each group when $80 \%$ power is anticipated with a hypothesis of determination of $10 \%$ difference between the akathisia rates $\left(\mathrm{p}^{2}-\mathrm{p}^{1}<10 \%\right)$, at 0.05 level of significance. We included 300 patients (154 SIG and 146 BIG) in our study.
Table 1 The Prince Henry Hospital akathisia rating scale*

OBJECTIVE RATINGS: (ratings by observer)

I. Sitting

1. Semipurposeful/purposeless leg/feet movement $0 \quad 1 \quad 2 \quad 3$

2. Semipurposeful hand/arm movements $\quad 0 \quad 1 \quad 2 \quad 3$

3. Shifting body position in chair $\quad 0 \quad 1 \quad 2 \quad 3$

4. Inability to remain seated $\quad 0 \quad 1 \quad 23$

II. Standing

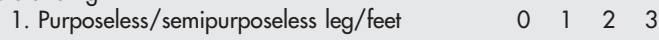
movements

2. Shifting weight from foot-to-foot and/or walking on spot

3. Inability to remain standing on one spot

$\begin{array}{llll}0 & 1 & 2 & 3\end{array}$ (walking or pacing)

Sum Score

SUBJECTIVE RATINGS: (three questions were asked)

1. Do you feel restless, or urge to move, especially $0 \quad \begin{array}{llll}0 & 2 & 3\end{array}$ in the legs?

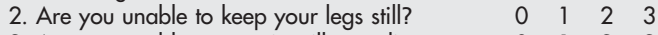

3. Are you unable to remain still, standing or $\quad \begin{array}{lllll}0 & 1 & 2 & 3\end{array}$ sitting?

Key: 0-3: absent, mild, moderate, severe

0 -absent

1 -mild and present some of time

2 -mild and present most of the time or severe and

present some of the time

3 -severe and present all the time

Sum Score

Total Score

*Reprinted from Biological Psychiatry, 35, ${ }^{4}$ Sachdev P. A rating scale for acute drug-induced akathisia: development, reliability, and validity, pp. 270-1, Copyright (1994) with permission from Society of Biological Psychiatry.

Global rating (by rater): 0 -absent; 1-mild; 2-moderate; 3-severe.

\section{RESULTS}

During the six month study period 320 eligible patients with headache and/or nausea/vomiting underwent evaluation for akathisia. Ten patients were excluded from the study because of inadequate records and five patients were excluded as they were taking medications listed in the exclusion criteria. Another five patients were excluded as they left the ED against medical advice.

Of the 300 patients randomised to the BIG and SIG groups, $151(50.3 \%)$ presented to the ED with nausea/vomiting, 108 $(36 \%)$ with headache, and 41 (13.7\%) with headache and nausea/vomiting. The demographic characteristics of the 300 patients are shown in table 2. Characteristics of the BIG and SIG groups were comparable.

In total 45 patients experienced akathisia (table 3). Comparing the two groups, 5.8\% (9/154) patients in the SIG group and $24.7 \%(36 / 146)$ patients in the BIG group had akathisia ( $\mathrm{p}<0.001$, OR 5.273, 95\% CI 2.43 to 11.403$)$.

Forty three patients (14.3\%) experienced akathisia in the first 15 minutes after metoclopramide infusions: $24(8 \%)$ in

Table 2 Patient characteristics

\begin{tabular}{|c|c|c|c|}
\hline & \multicolumn{2}{|l|}{ Group* } & \multirow[b]{2}{*}{ p value } \\
\hline & BIG $(n=146)$ & SIG $(n=154)$ & \\
\hline Age in years (mean (SD)) & 42.67 (17.10) & $39.94(16.30)$ & 0.157 \\
\hline Females & 115 & 120 & 0.859 \\
\hline $\begin{array}{l}\text { Weight in } \mathrm{kg} \text { (mean (SD)) } \\
\text { Indication }\end{array}$ & $66.99(12.29)$ & $65.71(12.92)$ & 0.380 \\
\hline Headache & 56 & 52 & \\
\hline Nausea/vomiting & 74 & 77 & \\
\hline $\begin{array}{l}\text { Headache+nausea/ } \\
\text { vomiting }\end{array}$ & 16 & 25 & \\
\hline
\end{tabular}

${ }^{*} \mathrm{BIG}, 10 \mathrm{mg}$ metoclopramide as intravenous bolus infusion over two minutes; SIG, $10 \mathrm{mg}$ metoclopramide as slow intravenous infusion over 15 minutes. 


\begin{tabular}{|c|c|c|c|}
\hline \multirow[b]{2}{*}{ Groupt } & \multicolumn{2}{|c|}{ Akathisia (n (\%)) } & \multirow[b]{2}{*}{ Total (n (\%)) } \\
\hline & No & Yes & \\
\hline SIG & 145 (94.2) & $9(5.8)$ & $154(100)$ \\
\hline BIG & $110(75.3)$ & $36(24.7)$ & $146(100)$ \\
\hline Total & $255(85.0)$ & 45 (15) & $300(100)$ \\
\hline \multicolumn{4}{|c|}{$\begin{array}{l}\text { *Fisher's test: } p<0.001 \text {. } \\
\text { †BIG, } 10 \mathrm{mg} \text { metoclopramide as intravenous bolus infusion } \\
\text { over two minutes; SIG, } 10 \mathrm{mg} \text { metoclopramide as slow } \\
\text { intravenous infusion over } 15 \text { minutes. }\end{array}$} \\
\hline
\end{tabular}

the first five minutes (of whom two patients $(0.6 \%)$ were in the SIG group) and $19(6.3 \%)$ between five and 15 minutes (of whom four patients (1.3\%) were in the SIG group). One patient $(2.2 \%)$ had akathisia between 15 and 30 minutes and another patient between 30 and 60 minutes (both patients were in the SIG group). There was no statistically significant association between the infusion rate and time of onset of akathisia (Fisher, $p=0.071$ and $p=1.00$, respectively, for incidence of akathisia at 5 minutes and 15 minutes in both groups).

Severe akathisia was observed in $13 / 45$ patients with akathisia $(28.8 \%)$. The incidence of severe akathisia was significantly higher in the BIG group $(30.5 \%, 11 / 36)$ compared with the SIG group $(22.2 \%, 2 / 9), p=0.009$. Sixteen patients experienced moderate akathisia of whom $75 \%(12 / 16)$ were in the BIG group and 15 patients had mild akathisia of whom $86.6 \%$ (13/15) were in the BIG group.

Metoclopramide successfully relieved the presenting symptom of $137 / 146$ patients (90.8\%) in the BIG group and of $139 /$ 154 patients $(90.2 \%)$ in the SIG group. There was no statistically significant difference between the two groups with regard to relief of presenting symptom(s) (Fisher, $\mathrm{p}=0.272$ ).

Although 35/45 of the patients with akathisia were female, there was no statistically significant relation between akathisia incidence and sex. There were also no statistically significant relations between akathisia incidence and the other demographic characteristics such as age, weight, previous diseases (diabetes, hypertension) in medical history, smoking, alcohol consumption, history of allergies, and history of medications (calcium channel blocking agents, $\beta$ receptor blocking agents, $\mathrm{H}_{2}$ blocking agents, or digoxin). None of the other side effects due to metoclopramide infusion observed in both the groups were statistically significant.

\section{DISCUSSION}

\section{Akathisia incidence}

Akathisia incidence due to medications varies in the literature. $^{8}{ }^{15} 16-18$ We achieved a significantly lower rate of akathisia by slowing down the rate of infusion of metoclopramide. A recent study reported the akathisia incidence as $3.5 \%$ in patients treated with metoclopramide alone. ${ }^{15}$ The main purpose of that study was not to investigate the akathisia incidence but to compare the effects of metoclopramide, meperidine, and their combination in the treatment of patients with recent onset primary headache. There was no comparison of rates of infusion of metoclopramide with regard to akathisia, and instead of the akathisia scale the observers used a yes/no questionnaire. In another study, a $12 \%$ rate of akathisia incidence was reported with a slow intravenous infusion of $10 \mathrm{mg}$ metoclopramide, but there was no comparison with bolus infusion. ${ }^{16}$ Miller and Jankovic stated that study design, form of the preparation, or long term medication use might account for the differences in the reported rates. ${ }^{7}$ It has been suggested that akathisia symptoms may occur just after the intravenous metoclopramide infusion and resolve quickly. Hence, in the clinical setting, unless clinicians closely observe patients for akathisia, the symptoms may be easily missed or underdiagnosed, especially in a busy ED. In our study we assessed the patients at zero, 5, 15, 30, and 60 minutes in the ED. This gave us the chance to observe closely, early in the treatment, the time of onset of the side effects. However, both the patients who participated in the study and the physicians who enrolled them complained about the study protocol as both groups found the close observation (five times in one hour) difficult.

\section{Severe akathisia}

We also found that the incidence of severe akathisia correlated with bolus infusion of metoclopramide $(p=$ 0.009 ). The lower incidence of akathisia reported in previous studies might have been the result of slowing down the infusion rate, as in our study. The mechanism by which akathisia occurs following metoclopramide infusion is still theoretical. Further studies correlating akathisia scores with serum metoclopramide levels may help to identify this mechanism.

We conclude that slow infusion is better and as of now report the lowest akathisia rate $(5.4 \%$ for slow infusion group, $\mathrm{p}<0.001$ ) in the literature.

\section{Onset time}

The onset time and duration of symptoms of akathisia were shorter than we have reported before. ${ }^{15}$ Twelve of the 24 patients who experienced akathisia at five minutes had no symptoms at 30 minutes. In eight of the 19 patients had akathisia at between five and 15 minutes, the symptoms disappeared without any medication at 30 minutes. We could not find any data regarding onset time of akathisia symptoms following intravenous metoclopramide infusion in previous studies. Most of the previous research was conducted in clinical settings other than the ED, and several of these basically investigated the side effects of oral metoclopramide treatment of various durations..$^{7}{ }^{18}$ Bateman et al found that the incidence of akathisia incidence in patients treated with oral metoclopramide was related to peak plasma concentration of metoclopramide (over $100 \mathrm{ng} / \mathrm{dl}$ ). They suggested that the route of administration is of major importance when determining the action of this drug. ${ }^{8}$

\section{Efficacy}

It has been reported that the most commonly used medications for parenteral treatment of isolated benign headache in EDs in the USA are meperidine $(30.0 \%)$, ketorolac $(21.4 \%)$, and prochlorperazine (16.7\%). ${ }^{19}$ Antiemetics may especially be useful for the resolution of headache and there is a trend suggesting the superiority of antiemetics. ${ }^{15} 20$ Metoclopramide, which is a benzamide antiemetic, is widely used in EDs in Turkey in the treatment of patients with isolated benign headache and/or nausea and vomiting. ${ }^{15} \mathrm{~A}$ limited number of studies have investigated the incidence of akathisia following metoclopramide infusion in patients with nausea/vomiting and headache..$^{15} 1618$ In a study comparing the efficacy of prochlorperazine with metoclopramide and placebo, Coppola et al reported that prochlorperazine had a significantly higher rate of efficacy in patients with migrainetype headache $\left(82 \%, 46 \%\right.$, and $29 \%$, respectively). ${ }^{4}$ On the other hand, Braude et al concluded that intravenous metoclopramide $(10 \mathrm{mg}$ ) and droperidol ( $1.25 \mathrm{mg}$ ) were significantly better for the control of nausea and vomiting in an unselected ED population than prochlorperazine or intravenous fluids alone. ${ }^{18}$ In our study, metoclopramide was an effective agent regardless of the route of administration in patients with nausea (143/151, 94.7\%), headache (95/108 patients, $87.9 \%)$, and nausea/headache (38/41 patients, $92.7 \%$ ). 


\section{Limitations of the study and future directions}

Our study has several limitations. Firstly, although data were collected and the patients rated by different physicians, we did not determine the interrater reliability for both data collection and rating of patients on the PHH akathisia scale. The physicians who recorded the data and rated the patients were trained in data collection, the PHH akathisia scale, clinical diagnosis, and management of akathisia in a two hour course prior to patient enrolment. However, interrater reliability for and validity of the $\mathrm{PHH}$ akathisia scale was examined in relation to the Barnes rating scale for akathisia (Barnes scale) in the original study by Sachdev. ${ }^{14}$ The kappa coefficient was calculated for each item and the global rating by two raters and all correlations were highly significant $(p<0.001)$. The correlation between the global akathisia ratings on the two scales was significant for both rater 1 $(0.84)$ and rater $2(0.86)$ (two tailed $t$ test, $\mathrm{p}<0.01$ ). Secondly, we used a yes/no questionnaire instead of a visual analogue scale to assess the severity of headache, nausea, and vomiting before and after metoclopramide infusion. Thirdly, we used a multichoice question for determining patient satisfaction rather than ideally a scale. Hence we could not statistically analyse the difference between the groups with regard to patient satisfaction. Finally, the patients were not observed for longer than an hour so we could not record how long the akathisia symptoms lasted and which rescue medications were needed.

These limitations did not directly influence the objective and results of the study. However, they restricted our discussion of patient satisfaction, successful treatment of akathisia, and the efficacy of metoclopramide infusion treatment in patients with headache and/or nausea/vomiting.

\section{CONCLUSIONS}

We suggest that slowing the infusion rate of metoclopramide is an effective strategy for reducing the incidence of akathisia in patients with headache and/or nausea/vomiting. Further clinical trials are needed to explain the mechanism of and relation between the incidence of akathisia and metoclopramide infusion rates.

\section{AUTHORS' CONTRIBUTIONS}

IP, RA, and MP conceived the study and designed the trial. IP and RA supervised the conduct of the trial and data collection. IP, MC, MP, $\mathrm{BE}, \mathrm{MG}, \mathrm{MS}$, and SK contributed to data collection. RA and MP provided statistical advice on study design and analysed the data; IP presented the data at the first World Congress of Emergency and Military Contigency Medicine, 3-8 June 2002, Kemer, Turkey. IP, RA, and MP drafted the manuscript. IP and RA revised the manuscript and all authors contributed substantially to its revision. IP takes responsibility for the paper as a whole.

\section{Authors' affiliations}

I Parlak, R Atilla, M Cicek, B Erdur, M Guryay, M Sever, S Karaduman, Dokuz Eylul University Hospital, Department of Emergency Medicine, Inciralti, Izmir, Turkey

M Parlak, Dokuz Eylul University Hospital, Department of

Anesthesiology, Inciralti, Izmir, Turkey

Competing interests: none declared

\section{REFERENCES}

1 Van Harten PN. [Drug-induced akathisia]. Ned Tijdschr Geneeskd 2002;146:110-14.

2 Mohr $\mathbf{P}$, Voavka J. Ladislav Haskovec and akathisia: 100th anniversary. Br J Psychiatry 2002; 181:537.

3 Bell R, Montoya D, Shuaib A, et al. A comparative trial of three agents in the treatment of acute migraine headache. Ann Emerg Med 1990;19:1079-82.

4 Coppola M, Yealy DM, Leibold RA. Randomized, placebo-controlled evaluation of prochlorperazine versus metoclopramide for emergency department treatment of migraine headache. Ann Emerg Med 1995;26:541-6.

5 Ellis GL, Delaney J, DeHart DA, et al. The efficacy of metoclopramide in the treatment of migraine headache. Ann Emerg Med 1993;22:191-5.

6 Tek DS, McClellan DS, Olshaker JS, et al. A prospective, double-blind study of metoclopramide hydrochloride for the control of migraine in the emergency department. Ann Emerg Med 1990;19:1083-7.

7 Miller LG, Jankovic J. Metoclopramide-induced movement disorders. Clinical findings with a review of the literature. Arch Intern Med 1989;149:2486-92.

8 Bateman DN, Kahn C, Mashiter K, et al. Pharmacokinetic and concentrationeffect studies with intravenous metoclopramide. Br J Clin Pharmacol 1978;6:401-7

9 Caldwell C, Rains G, McKiterick K. An unusual reaction to preoperative metoclopramide. Anesthesiology 1987;67:854-5.

10 Collins RW, Jones JB, Walthall JDH, et al. Intravenous administration of prochlorperazine by 15 -minute infusion versus 2 -minute bolus does not affect the incidence of akathisia: a prospective, randomized, controlled trial. Ann Emerg Med 2001;38:491-6.

11 Chow LY, Chung D, Leung V, et al. Suicide attempt due to metoclopramideinduced akathisia. Int J Clin Pract 1997;51:330-1.

12 Drake RE, Ehrlich J. Suicide attempts associated with akathisia. Am J Psychiatry 1985; 142:499-501.

13 LaGorio J, Thompson VA, Sternberg D, et al. Akathisia and anesthesia: refusal of surgery after the administration of metoclopramide. Anesth Analg 1998:87:224-7.

14 Sachdev P. A rating scale for acute drug-induced akathisia: development, reliability, and validity. Biol Psychiatry 1994;35:263-71.

15 Cicek M, Karcioglu O, Parlak I, et al. Prospective, randomized, double blind, controlled comparison of metoclopramide and pethidine in the emergency treatment of acute primary vascular and tension type headache episodes. Emerg Med J 2004;21:323-6.

16 Seviour CM, Harrison DW, Abu-Laban RB. Incidence of akathisia from intravenous metoclopramide for migraine headache. Acad Emerg Med 2000;7:536.

17 Ganzini L, Casey DE, Hoffman WF, et al. The prevalence of metoclopramideinduced tardive dyskinesia and acute extrapyramidal movement disorders. Arch Intern Med 1993;153:1469-75.

18 Braude DA, Andrews J, Soliz T, et al. Prospective comparison of three common anti-emetic medications in the emergency. An interim analysis. Acad Emerg Med 2000;7:445.

19 Vinson DR. Treatment patterns of isolated benign headache in US emergency departments. Ann Emerg Med 2002;39:215-22.

20 Morgenstern LB, Huber JC, Luna-Gonzales $\mathrm{H}$, et al. Headache in the emergency department. Headache 2001;41:537-41. 